\title{
Antimicrobial resistance: concerns of healthcare providers and people with CF
}

Wendy Bullington ${ }^{1}$, Sarah Hempstead ${ }^{2}$, Alan R. Smyth ${ }^{3}$, Pavel Drevinek ${ }^{4}$, Lisa Saiman ${ }^{5}$, Valerie J. Waters $^{6}$, Scott C. Bell ${ }^{7}$, Donald R. VanDevanter ${ }^{8}$, Patrick A. Flume ${ }^{9}$, Stuart Elborn ${ }^{10}$, Marianne Muhlebach ${ }^{11}$ on behalf of the Antimicrobial Resistance International Working Group in Cystic Fibrosis

${ }^{1}$ Department of Pharmacy Services, Medical University of South Carolina, Charleston, SC, USA. bullingw@musc.edu.

${ }^{2}$ Cystic Fibrosis Foundation, Bethesda, MD, USA. shempstead@cff.org

${ }^{3}$ Division of Child Health, Obstetrics \& Gynaecology, University of Nottingham, Nottingham, United Kingdom. Alan.Smyth@nottingham.ac.uk

${ }^{4}$ Departments of Medical Microbiology Motol University Hospital and $2^{\text {nd }}$ Faculty of Medicine, Charles University, Prague, Czech Republic. pavel.drevinek@lfmotol.cuni.cz

${ }^{5}$ Department of Pediatrics, Columbia University, New York, New York, USA.

\section{Is5@cumc.columbia.edu}

6 Division of Infectious Diseases, Department of Pediatrics, The Hospital for Sick Children, University of Toronto, Toronto, Canada. Valerie.Waters@sickkids.ca

${ }^{7}$ Department of Thoracic Medicine, The Prince Charles Hospital and QIMR Berghofer Medical Research Institute, Brisbane, Australia. scott.bell@health.qld.gov.au

${ }^{8}$ Department of Pediatrics, Case Western Reserve University School of Medicine, Cleveland, $\mathrm{OH}$, USA. drv15@case.edu

${ }^{9}$ Departments of Medicine and Pediatrics, Medical University of South Carolina, Charleston, SC, USA. flumepa@musc.edu

${ }^{10}$ Imperial College and Royal Brompton Hospital, London and Queen's University Belfast, United Kingdom.s.elborn@qub.ac.uk

${ }^{11}$ Department of Pediatrics, University of North Carolina, Chapel Hill, NC, USA. marianne muhlebach@med.unc.edu 


\section{Corresponding author/requests for reprints:}

Dr. Wendy M. Bullington

Department of Pharmacy Services

150 Ashley Avenue, Room 504, MSC 584

Charleston, SC 29425 USA

E-mail: bullingw@musc.edu

Word count: 3099 words (abstract 173 words)

Key words: cystic fibrosis, antimicrobial resistance, survey 


\begin{abstract}
Background: Chronic lung infections and their treatment pose risks for the development of antimicrobial resistance (AMR) in people with cystic fibrosis (PWCF). In this study, we evaluated the attitudes of healthcare providers' (HCP) and PWCF or their parents' toward AMR within the international CF community.

Methods: HCP and PWCF identified through listservs and CF-related organizations were asked to complete an AMR centered survey, with additional questions on antimicrobial stewardship (AMS) for HCP. Descriptive analyses are reported.

Results: The responding 443 HCP and 464 PWCF/Parents were from 30 and 25 countries, respectively. Sixty-two percent of HCP and $56 \%$ of PWCF stated they were "very concerned" about AMR, with Pseudomonas spp. and Burkholderia spp. considered the most concerning organisms for both HCP and PWCF/Parents. Non-tuberculous mycobacteria were of greater concern to HCP compared to PWCF/Parents. There was a discrepancy regarding AMR education to PWCF, with $80 \%$ of HCP stating having discussed this with PWCF/Parents, but only $50 \%$ of PWCF recalling such discussions.

Conclusion: These results highlight that AMR is relevant to CF HCP and PWCF internationally, indicating that educational tools and research are warranted.
\end{abstract}




\section{INTRODUCTION}

Antimicrobial resistance (AMR) broadly defined, refers to the inability of antimicrobials to effectively inhibit the growth of or kill a given microorganism. ${ }^{1}$ AMR is increasing among microbial opportunists, which is a major concern for healthcare providers (HCP), patients, and the general public. In CF, the emergence of AMR is multi-factorial due to chronic lung infections with bacterial adaptation and to the use of chronic suppressive antibiotic therapy with additional antibiotics for exacerbations. ${ }^{2,3,4,5}$ In CF care centers in the USA, proportions of people with CF (PWCF) with multi-drug resistant (MDR) Pseudomonas aeruginosa isolated from respiratory cultures has increased over time and is now $25-30 \% .{ }^{5}$ Yet, greater use of antibiotics has been historically associated with better patient outcomes in CF, making the concept of using less antibiotics to decrease AMR challenging and situation dependent.

The increasing prevalence of AMR may require re-addressing antibiotic use more carefully in CF. ${ }^{9}$ Measures to address AMR have led to the development of hospital and community antimicrobial stewardship programs (AMS) around the world. ${ }^{10,11}$ The goals of AMS programs are to improve the treatment of infections by assisting in selection of antimicrobials, prescribing proper doses and durations, and monitoring for toxicity and other adverse effects. ${ }^{12}$

The AMR in CF International Working Group (funded by European CF Society, U.S. CF Foundation, CF Trust, CF Australia, and CF Canada) was created to inform the CF community on important issues related to AMR. ${ }^{2,13}$ In order to design educational and/or treatment related interventions, it is important to understand current knowledge and attitudes of the stakeholders regarding AMR. The aim of this study was to assess the current knowledge, concerns, and educational needs of the CF community (i.e. PWCF or their parents, termed here PWCF/Parents) and HCP with regards to AMR. Further, we surveyed HCP about AMS resources in their practice environment and attitudes towards AMS. 


\section{METHODS}

A subgroup of the International Working Group developed a survey to explore five topics: 1) knowledge of AMR; 2) level of concern and perceived risks; 3) perceived consequences of AMR; 4) desired information; 5) preferred information sources. HCP were asked an additional six questions related to AMS. The initial survey was pilot tested by ten internationally recruited HCP and eight PWCF from three CF centers. After modifications based on feed-back from pilot testers, the surveys (Survey Monkey) were distributed internationally to HCP and PWCF/Parents in July 2018 through the Cystic Fibrosis Foundation, the European Cystic Fibrosis Society, CF Canada, and CF Australia and were open for one month. Details of distribution and the complete survey are available in an online Supplement. Percentages were calculated as proportion of valid answers to each question. Data for sub-groups of PWCF/Parents are shown when relevant differences were noted. Descriptive statistics were performed using MS Excel and JMP®Pro 12.0.1 (SAS Institute).

\section{RESULTS}

We received responses from HCP $(n=443)$ with various clinical roles from 30 countries, including 78\% where English was the respondent's first language (Figure 1A). Providers were involved in

pediatric care (44\%), adult care (33\%), or both age groups (23\%). Among physicians, the majority (71\%) were respirologists/pulmonologists, $5 \%$ were in infectious disease/infection controlepidemiology, and 17\% were classified as "other," which included 11 general practitioners. Time engaged in the care of PWCF ranged from $<1$ year to $>20$ years (most common, $1-5$ years $(28 \%))$ with an even distribution across the continuum. CF center sizes were evenly distributed in size, with numbers of PWCF ranging in four categories from $<50$ to $>200$; the majority reported being in centers with a size of 101-200 PWCF. Among the PWCF/Parents $(n=464)$ who responded, 25 countries were represented and 56\% lived in an English-speaking country. The majority were parents of children with CF (Figure 1B). Response rates for survey completion was significantly higher for HCP than PWCF/Parents (mean \pm SEM. $89.8 \pm 0.02 \%$ vs. $73.4 \pm 0.02 \%$, 
$\mathrm{p}<0.001)$. Response rates did not differ across topics in either group of respondents.

\section{Knowledge of $A M R$}

The term antibiotic and/or antimicrobial resistance was known to all but one social worker in the HCP group ( 100\%) and to $96 \%$ of PWCF/Parents. Using four vignettes and allowing more than one response, $88 \%$ of $\mathrm{HCP}$ and $66 \%$ of PWCF/Parents selected microbiology-laboratory based definitions of AMR and $61 \%$ of $\mathrm{HCP}$ and $81 \%$ of PWCF/Parents chose vignettes that indicated patient response to therapy as a clinical definition. Respondents were also asked for short descriptors of the term "antimicrobial resistance". Descriptor responses (more than one answer allowed) were categorized into the following areas: 1) bacterial mechanisms, chosen by $66 \% \mathrm{HCP}$ and $57 \%$ PWCF/Families, with an additional $15 \%$ of HCP adding specific microbiology laboratory definitions 2) antibiotic/clinical outcomes-related definitions provided by $4 \%$ and $14 \%$ of HCP and PWCF/Parents, respectively, and 3) definitions that implicated AMR as a host-related event were given by $1 \%$ of HCP and $9 \%$ of PWCF/Parents. Nine percent of HCP and $5 \%$ of PWCF/Parents provided incomplete responses. Further, $7 \%$ of PWCF/Parents were unable to provide their own definition; these rates did not differ by primary language.

\section{Level of concern and perceived risks}

A majority of HCP (62\%) and PWCF/Parents (56\%) rated their concern about AMR as "very concerned". In contrast, a single HCP and $1 \%$ of PWCF/Parents stated not being concerned about AMR. The estimated frequency of AMR in PWCF ranged from "some of the time" (48\% HCW and 53\% PWCF/Parents) to "most of the time" (42\% HCW and 38\% PWCF/Parents) to "all of the time" ( $9 \%$ in each group).

A majority of respondents in each group perceived AMR risk to be different in some individuals with CF compared to others. Table 1 summarizes which situations (more than one answer allowed) or locations (single, ranked answers) were perceived as relevant. When ranking risk factors, a majority of HCP (73\%) chose frequent intravenous or oral antibiotics (i.e., frequency < 
every 2 months) over prolonged oral antibiotics (15\%), >2 inpatient admissions per year (6\%), and older age (6\%). Among PWCF/Parents the majority (57\%) chose frequent antibiotics over prolonged oral antibiotics (21\%), >2 inpatient admissions per year (12\%), and older age (10\%).

Pseudomonas spp. (69\%, 69\%) and Burkholderia spp. (55\%, 43\%) were most concerning to HCP and PWCF/Parents, followed in frequency $(52 \%)$ by non-tuberculosis mycobacteria (NTM) among HCP. Interestingly, HCP were less concerned than PWCF/Parents about Staphylococcus aureus in general (12\% vs. $28 \%$ ), but concerns for methicillin-resistant Staphylococcus aureus (MRSA) doubled in both groups (25\% vs. $47 \%$ ); MRSA infection is often highlighted in lay media and may lead to worse outcomes in PWCF. ${ }^{14,15}$ Other organisms and distributions by country/continent are shown in Table 2.

\section{Consequences of AMR}

Congruence was high between HCP and PWCF/Parents for perceived consequences of AMR, including the influence of AMR on the choice of antibiotics $(89 \%$ of HCP and $81 \%$ of PWCF/ Parents agree) and AMR adversely affecting life expectancy (88\% of HCP and $92 \%$ of PWCF agree). There was less congruence for "AMR affects response to antibiotics" (94\% of HCP and $78 \%$ of PWCF/Parents). We also asked whether "PWCF/Parents did not complete their antibiotic course as prescribed because of concerns of AMR". Among HCP, $86 \%$ did not think this occurred, compared to $77 \%$ of PWCF/Parents who stated never having not completed therapy. Parents answered "never" in $83 \%$ of cases compared to $75 \%$ of adults vs. $57 \%$ of adolescents with CF. Additionally, $16 \%$ of PWCF/Parents stated they rarely had not completed therapy, $2 \%$ stated not completing therapy often, and $1 \%(n=3)$ responded "always".

\section{Frequency and type of AMR education}

Results regarding the estimated frequency of information provided to PWCF/Parents showed a discrepancy between groups in response rate (HCP 89\% vs. PWCF/F 73\%, p<0.001), with equal distribution of missing answers within the sub-groups of PWCF/Parents. The HCP 
estimated providing AMR education to PWCF/Parents at a frequency of $38 \%, 45 \%$, and $17 \%$ once a year, every other visit, or every visit, respectively. Among responding PWCF/Parents, only $50 \%$ stated that they were given information about AMR, yet this differed by subgroups with $64 \%$ of adults and $63 \%$ of adolescents with CF compared to $39 \%$ of parents remembering this. Only $35 \%$ of PWCF/Parents provided an estimated frequency of AMR discussions, with once a year (61\%), every other visit (32\%) and at every clinic visit (7\%) and those estimates were similar across PWCF/Parents subgroups.

Fifty percent of HCP provided free text answers discussing the context in which they provided AMR education. The most common situations were related to antibiotic treatment or to hospital admissions (jointly 66\%), 17\% discussed AMR when new or newly resistant bacteria were isolated, $9 \%$ discussed AMR as part of routine education or when prompted by PWCF/Parents, and $8 \%$ had various/other responses. PWCF/Parents stated that the following information was included (>1 option): definition of AMR (61\%), infection control measures (61\%), and antibiotic overuse (38\%).

Responses about current resources PWCF/Parents utilized to obtain information on AMR were similar between the groups and showed that the preferred source of information was communication from their CF team (Figure 2). Open comments further suggested that flyers and handouts would be desired followed by national or international CF specific websites.

\section{Expectations and attitudes towards Antimicrobial Stewardship among HCP}

Response rate for the AMS questions was $86 \%$. Available resources reported by respondents were pharmacists (69\%), infectious disease consultation (65\%), and local written exacerbation guidelines (55\%). Fewer had a formal AMS program (39\%), pre-approval process for restricted antibiotics (41\%), and post-prescription feedback and audit (8\%) in their institution. Most respondents perceived that choosing and dosing antibiotics appropriately and reducing AMR were the main goals of AMS (Figure 3). When asked whether they believed that AMS 
programs preserved the activity of antibiotics, a higher proportion thought this to be true in people without CF $(71 \%)$ vs. people with CF $(55 \%)$. About a third stated that they didn't know whether AMS was helpful in those without CF (26\%) or those with CF (34\%). The effect of AMS in managing CF exacerbations was considered to be "a large impact" by $32 \%$ of HCP and "to have some impact" by $58 \%$. Ten percent thought AMS would have "minimal to no impact". Stewardship activities considered useful during CF exacerbations included: choice of antibiotics (83\%), duration of antibiotics (78\%), dose of antibiotics (68\%), therapeutic drug monitoring (63\%), minimizing drug interactions (53\%), and avoiding toxicity (50\%). Nine percent of respondents stated that they did not know the benefit of AMS during exacerbations.

\section{DISCUSSION}

A survey of over $900 \mathrm{HCP}$ and PCWF/Parents from over 30 countries illustrated that AMR was thought to be important to care and that responses between HCP and PWCF/Parents were largely congruent. A majority of HCPs and PWCF/Parents were "very concerned" about AMR and listed frequent use of antibiotics as the most important risk factor. A high proportion of HCP and PWCF/Parents felt that AMR affected life expectancy. Concerns about person-to-person transmission of infection as a source for AMR was listed by over $50 \%$ of respondents (PWCF/Parents > HCP) (Table 1). This is an important finding as such concerns may affect the likelihood of PWCF attending appointments, which was mentioned in open text by some PWCF/Parents. This also stresses the need for ongoing focus on infection prevention, education, and control measures. Interestingly, most HCPs believed they discussed AMR with PWCF/Parents; however, fewer PWCF/Parents remembered receiving this information, especially parents. This highlights the need for HCP to provide better means of education, e.g. written handouts.

A recent priority-setting exercise, involving both the lay and clinical CF communities, identified the negative effects of antibiotics (including development of antimicrobial resistance) as one of 
the top research priorities. ${ }^{16,17}$ This is consistent with answers given by PWCF in surveys conducted through the U.S. CF Foundation, where PWCF listed respiratory microorganism detection and treatment as the top research priority. ${ }^{18,19}$ However, a central paradox of CF care is that, whilst antibiotic resistance is prevalent, there appears to be little relationship between the results of antibiotic susceptibility testing and clinical response to antibiotic treatment.2,20,21 Such a paradox may be compounded by historical data from U.S. CF centers showing better pulmonary outcomes with higher intravenous antibiotic use $;^{7,8,22}$ this was not seen in a more recent study using the UK CF Patient Registry. ${ }^{23}$

This study has limitations. Given the self-selection of respondents, they may be more aware of AMR and AMS compared to other people in professional and lay communities. The English language survey may have limited response rate among non-native English speakers in Europe; however, this did not seem to affect results by those who took the survey. Additionally, surveys were subject to recall bias and responses were restricted to the questions asked on the survey. Although the survey included 27 and 23 questions for HCP and PWCF/Parents, respectively, the response rate did not decrease towards the later questions.

Our findings imply that "road testing" of specific approaches to patient education about AMR (such as infographics and podcasts) and looking at the effectiveness of different media (social media vs. written material from the CF center) would be of particular interest. Both HCPs and PWCF/Parents would like to see research that evaluates antibiotic therapy to maximize effectiveness and avoid the emergence of resistance. ${ }^{16,18,24}$ This may include measures to reduce the need for antibiotics, such as eradication strategies ${ }^{25,26}$ to prevent development of persistent infection, systematically evaluating the duration of antibiotics for exacerbations (STOP2 and Ped. STOP trial) ${ }^{27,28}$ and measures of infection prevention and control. ${ }^{29}$ There is a need to understand how microbiologic lab testing can best inform treatment decisions as current microbiology susceptibility methods of testing may not mimic CF airways growth conditions. 3031 
Other suggestions for AMS have recently been proposed, such as strengthening relationships between CF clinical and AMS teams, in order to consider implementing stewardship strategies. ${ }^{9}$

The disconnect between antibiotic strategies and clinical outcomes suggest that both basic science and translational approaches are needed. As an immediate direction, HCP should enhance their discussions about AMR during CF clinic visits to educate a broader audience given that PWCF/Parents want to learn about AMR from their providers.

\section{Acknowledgements:}

The authors would like to thank the Cystic Fibrosis Foundation for facilitating research through Community Voice, the European Cystic Fibrosis Foundation, CF Canada, and CF Australia for facilitating research through their patient communities to support this work. Additionally, we would like to thank all the adults with cystic fibrosis and family members across North America, Europe, and Australasia, who participated for sharing their insights.

The authors wish to acknowledge the work of the other members of the Antimicrobial Resistance in Cystic Fibrosis International Working Group: Shawn Aaron (Ottawa General Hospital, Ottawa, Canada), Pierre-Regis Burgel (Paris Descartes University, Paris, France), Catherine Byrnes (Auckland Hospital, Auckland, New Zealand), Rafael Canton (Hospital Universitario Ramón y Cajal, Madrid, Spain), Miquel Ekkelenkamp (University Medical Center Utrecht, Utrecht, Netherlands), Peter Gilligan (UNC Healthcare, Chapel Hill, NC, USA), Alison Holmes (Imperial College London, London, United Kingdom), Helle Krogh Johansen (University of Copenhagen, Copenhagen, Denmark), Barbara Kahl (University Hospital Münster, Münster, Germany), Timothy Kidd (The University of Queensland, Brisbane, Australia), John LiPuma (University of Michigan, Ann Arbor, MI, USA), Holly Maples (University of Arkansas for Medical Sciences, Little Rock, USA), Stacey Martiniano (Children's Hospital Colorado, Aurora, USA), Susanna McColley (Children's Hospital of Chicago, Chicago, USA), Andrew Morris (University Health Netwok, Sinai Health System, Toronto, Canada), Michael Parkins (University of Calgary, Calgary, Canada), Felix Ratjen (Hospital for Sick Children, Toronto, Canada), Jason Roberts (Royal Brisbane and Women's Hospital, Brisbane, Australia), Anand Shah (Royal Brompton Hospital, London, UnitedKingdom), Pradeep Singh (University of Washington, Seattle, USA), Ranjani Somayaji (University of Calgary, Calgary, Canada), Giovanni Taccetti (Anna Meyer Children's University Hospital, Florence, Italy), Michael Tunney (Queen's University Belfast, Belfast, United Kingdom), Francoise Van Bambeke (Université catholique deLouvain, Brussels, Belgium), Kevin Winthrop (Oregon Health and Science University, Portland, USA), Edith Zemanick(Children's Hospital Colorado, Aurora, USA).

Drs. Elborn and Muhlebach and Ms. Hempstead have nothing to disclose.

Dr. Bullington reports a grant outside this work from the Cystic Fibrosis Foundation.

Dr. Smyth reports a grant and personal fees from Vertex, speaker honorariums and expenses from Teva and Novartis, and a patent on alkyl quinolones as biomarkers of pseudomonas aeruginosa infection outside of this work. 
Dr. Drevinek reports personal fees from Vertex, Proteostasis, and Corbus Pharmaceuticals outside of this work.

Dr. Walters reports grants from Gilead and an Astrazeneca consultancy outside of this work.

Dr. VanDevanter reports personal fees from AbbVie, Albumedix, AN2, Aradign, Armata, Arrevus, Calithera, Chiesi USA, Cipla, Cystic Fibrosis Foundation, Corbus, Eloxx, Enbiotix, Eveo, Galephar, Horizon, IBF, ICON Clinical Sciences, Ionis, Kala, Merck, Microbion, NDA, Protalix, PTC, Pulmocide, Recida, Savara, Vast, and Vertex outside of this work.

Dr. Flume reports grants outside of this submitted work from the European Cystic Fibrosis Society, the Cystic Fibrosis Foundation, Cystic Fibrosis Trust, Cystic Fibrosis Canada, Cystic Fibrosis Australia, Novartis, and Novoteris. Dr. Flume also receives personal fees from Insmed outside of this submitted work.

Dr. Bell reports grants and other support from Vertex, Galapagos, and AbbVie outside of this work. 


\section{Figure Legends}

Figure 1: Characteristics of Respondents for A) Health care providers (HCP) showing country of current work and their role in CF care; B) People with CF (PWCF) with country. Fourteen did not provide a response regarding their role/connection to $\mathrm{CF}$. Abbreviations: $\operatorname{Inf} \mathrm{Ctr}=$ Infection control/Hospital epidemiology. Inpt = inpatient; SW= Social worker

1

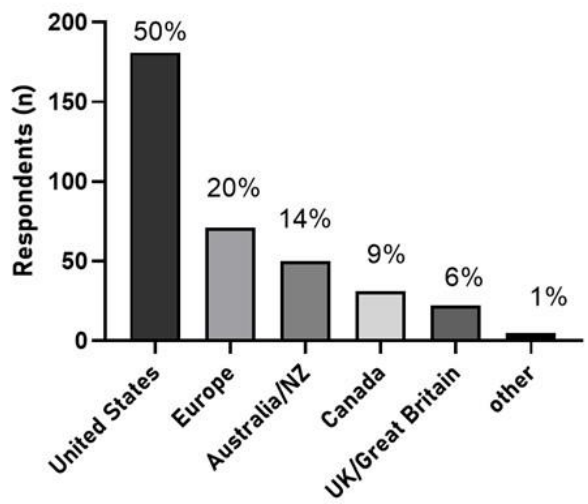

\begin{tabular}{lc}
\hline \multicolumn{1}{c}{ Role in CF care } & \% of respondents \\
\hline & 48 \\
Physician & 16 \\
CF nurse specialist & 10 \\
CF pharmacist & 9 \\
Physical- Resp. therapist & 7 \\
Other (self defined) & 10 \\
Other groups (Inf. Ctrl; inpt & \\
nurse; dietician, SW, Advanced & \\
care practioner) & \\
\hline
\end{tabular}




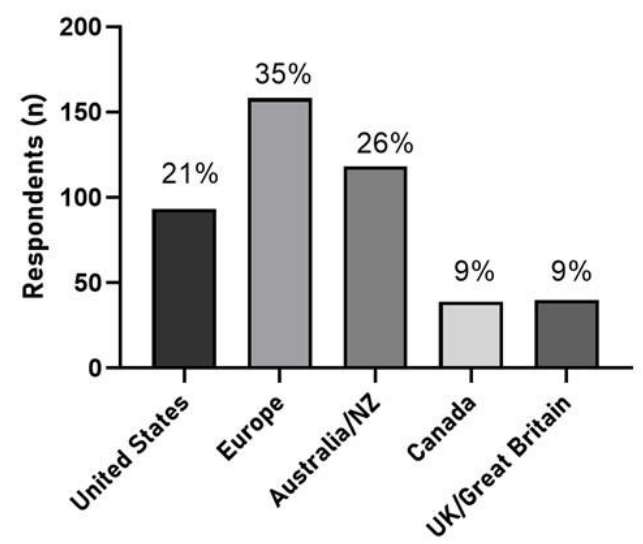

\begin{tabular}{cc}
\hline PWCF sub-group & $\%$ Respondents \\
\hline Parent of CF child & $57 \%$ of all respondents \\
Child is $0-3$ years & $21 \%$ of parents \\
Child is $4-6$ years & $17 \%$ \\
Child is $7-13$ years & $33 \%$ \\
Child is $14<18$ years & $28 \%$ \\
Adolescent (14 -18 yrs) w CF & $2 \%$ of all respondents \\
Adult with CF & $41 \%$ of all respondents \\
Adult $18-25$ years & $17 \%$ \\
Adult 26 - 35 years & $41 \%$ \\
Adult $>35$ years & $82 \%$ \\
\hline
\end{tabular}

Figure 2: Sources where HCP perceive PWCF get information about antimicrobial resistance; and where PWCF state they get their information about AMR. Respondents checked all that applied.

Figure 3: Healthcare provider responses to questions on the goals of antimicrobial stewardship in CF. Respondents were allowed to check more than one answer. 
Tables

Table 1: Perceived situational and location-specific risk factors by HCP and PWCF

\begin{tabular}{|c|c|c|c|c|c|}
\hline \multirow[t]{2}{*}{ Situational risk } & \multicolumn{2}{|c|}{$\begin{array}{c}\% \text { of } \\
\text { respondents }\end{array}$} & \multirow[t]{2}{*}{ Location-specific risk } & \multicolumn{2}{|c|}{$\begin{array}{c}\% \text { of } \\
\text { respondents }\end{array}$} \\
\hline & HCP & PWCF & & HCP & PWCF \\
\hline Cycled inhaled antibiotics & 31.5 & 29.3 & Home & 3.0 & 1.7 \\
\hline Country of residence & 28.5 & 21.4 & School or work & 1.8 & 6.4 \\
\hline $\begin{array}{l}\text { Frequent inpatient } \\
\text { antibiotics }\end{array}$ & 81.1 & 63.1 & $\begin{array}{l}\text { CF outpatient clinic } \\
\text { appointment }\end{array}$ & 28.2 & 22.0 \\
\hline Any hospitalization & 31.9 & 40.5 & Inpatient admissions & 53.1 & 47.0 \\
\hline $\begin{array}{l}\text { Frequent outpatient } \\
\text { antibiotics }\end{array}$ & 78.8 & 66.3 & Non-CF medical appointment & 7.2 & 9.0 \\
\hline $\begin{array}{l}\text { Same antibiotics within } 3 \\
\text { months }\end{array}$ & 54.9 & 48.4 & $\begin{array}{l}\text { Non-healthcare crowded } \\
\text { areas }\end{array}$ & 6.7 & 13.9 \\
\hline Transmission among PWCF & 51.4 & 56.6 & Other / no response & $<1$ & $<1$ \\
\hline CFTR mutation & $\begin{array}{r}16.9 \\
50\end{array}$ & 17.9 & & & \\
\hline
\end{tabular}

Table 1: 397 healthcare providers (HCP) and 341 people with CF (PWCF) responded to the situational risk questions. Respondents were able to choose more than one answer for situational risks. Responses to the location-specific risk questions came from $401 \mathrm{HCP}$ and 345 PWCF. Only one choice was allowed for this question. 
Table 2

HCP and PWCF concern by organism for AMR

\begin{tabular}{lcccccc}
\hline \multirow{2}{*}{ Organism } & \multicolumn{2}{c}{\begin{tabular}{c} 
Australia/New \\
\multicolumn{1}{c}{ Zealand $\mathrm{n}=58$}
\end{tabular}} & \multicolumn{2}{c}{ Europe } & \multicolumn{2}{c}{ USA/Canada } \\
& \multicolumn{2}{c}{ \% of respondents } & \multicolumn{2}{c}{ \% of respondents } & \multicolumn{2}{c}{ \% of respondents } \\
& HCP & PWCF & HCP & PWCF & HCP & PWCF \\
\hline Pseudomonas & $38(66)$ & $77(65)$ & $65(53)$ & $94(48)$ & $163(68)$ & $72(55)$ \\
Burkholderia & $28(48)$ & $41(35)$ & $63(52)$ & $55(28)$ & $115(48)$ & $56(42)$ \\
Staphylococcus & $5(9)$ & $30(25)$ & $11(9)$ & $38(19)$ & $32(13)$ & $30(23)$ \\
NTM & $32(55)$ & $25(21)$ & $55(45)$ & $41(2)$ & $114(48)$ & $31(23)$ \\
MRSA & $9(16)$ & $49(42)$ & $22(18)$ & $60(31)$ & $66(28)$ & $55(42)$ \\
Streptococcus & 0 & $13(11)$ & $1(1)$ & $18(9)$ & $5(2)$ & $19(14)$ \\
Stenotrophomonas & $3(5)$ & $8(7)$ & $12(10)$ & $10(5)$ & $31(13)$ & $7(5)$ \\
Aspergillus & $1(2)$ & $23(19)$ & $6(5)$ & $23(12)$ & $18(8)$ & $24(18)$ \\
Influenza virus & 0 & $12(10)$ & $1(1)$ & $5(3)$ & $3(1)$ & $12(9)$ \\
\hline
\end{tabular}

Table 2: Healthcare providers' (HCP) and people with cystic fibrosis (PWCF) level of concern by organism for antimicrobial resistance (AMR) shown by continent. Percentage of respondents (\%) by continent exceeds 100 as participants could choose up to two organisms. NTM - nontuberculous mycobacteria. MRSA - methicillin resistant Staphylococcus aureus. 


\section{REFERENCES}

${ }^{1}$ Waters VJ, Kidd TJ, Canton R, Ekkelenkamp MB, Johansen HK, LiPuma JJ, et al. Reconciling
antimicrobial susceptibility testing and clinical response in antimicrobial treatment of chronic cystic fibrosis lung infections. Clin Infect Dis 2019;69(10):1812-1816

${ }^{2}$ Somayaji R, Perkins MD, Shah A, Martiniano SL, Tunney MM, Kahle JS, et al. Antimicrobial susceptibility testing (AST) and associated clinical outcomes in individuals with cystic fibrosis: A systematic review. J Cyst Fibro 2019 Mar;18(2):236-243

${ }^{3}$ Kidd TJ, Canton R, Ekkelenkamp M, Johansen HK, Gilligan P, LiPuma JJ, et al. Defining antimicrobial resistance in cystic fibrosis. J Cyst Fibros 2018; 17:696-704

${ }^{4}$ Döring G, Flume P, Heijerman H, Elborn JS and Consensus Study Group Collaborators. Treatment of lung infection in patients with cystic fibrosis: current and future strategies. J Cyst Fibros 2012; 11(6):461-79.

${ }^{5}$ Cystic Fibrosis Foundation Patient Registry. 2017 Annual Data Report.

${ }^{6}$ Ramsey B, Smith AL, et al. Intermittent administration of inhaled tobramycin in patients with cystic fibrosis. Cystic Fibrosis Inhaled Tobramycin Study Group. N Engl J Med. 1999 Jan 7;340(1):23- 30 .

7 Johnson C, Butler SM, Konstan MW, Morgan W, Wohl ME. Factors influencing outcomes in cystic fibrosis: a center-based analysis. Chest 2003;123:20-7.

${ }^{8}$ Saiman L, Marshall BC, Mayer-Hamblett N, et al. Azithromycin in patients with cystic fibrosis chronically infected with Pseudomonas aeruginosa: a randomized controlled trial.

JAMA 2003;290:1749-1756

${ }^{9}$ Cogen JD, Kahl BC, Maples H, McColley SA, Roberts JA, Winthrop KL, Morris AM, Holmes A, Flume PA, VanDevanter DR, Waters V, Muhlebach MS, Elborn JS, Saiman L, Bell SC. Finding the relevance of antimicrobial stewardship for cystic fibrosis. J Cyst Fibr: published online Feb 29, 2020. https://doi.org/10.1016/.j.jcf.2020.02.012.

${ }^{10}$ https://www.cdc.gov/antibiotic-use/healthcare/implementation/core-elements.html. Accessed April 25, 2019

${ }^{11}$ https://eur-lex.europa.eu/legalcontent/EN/TXT/?uri=uriserv:OJ.C .2017.212.01.0001.01.ENG\&toC=OJ:C:2017:212:TOC, Accessed April 25, 2019

12 Barlam TF, Cosgrove SE, Abbo LM, MacDougall C, Schuetz AN, Septimus EJ, et al. Implementing an antibiotics stewardship program: guidelines by the Infectious Diseases Society of America and the Society for Healthcare Epidemiology of America. Clin Infect Dis 2016;62(10):e51e77

${ }^{13}$ Flume PA, Waters VJ, Bell SC, VanDevanter DR, Elborn JS. Antimicrobial resistance in cystic fibrosis: Does it matter? J Cys Fibros 2018:17(6):687-689 
${ }^{14}$ Bell SC, Flume PA. Treatment decisions for MRSA in patients with cystic fibrosis (CF): when is enough, enough? Thorax 2017:72:297-99

${ }^{15}$ Dasenbrook EC, Checkley W, Merlo CA, Konstan MW, Lechtzin N, Boyle MP. Association between respiratory tract methicillin-resistant Staphlococcus aureus and survival in cystic fibrosis. JAMA 2010; 303(23): 2386-92

${ }^{16}$ Rowbotham NJ, Smith S, Leighton PA, Rayner OC, Gathercole K, et al. The top 10 research priorities in cystic fibrosis developed by a partnership between people with CF and healthcare providers. Thorax 2018; 73(4): 388-90

${ }^{17}$ Hollin IL, Donaldson SH, Roman C, Aliaj E, Riva D, et al. Beyond the expected: Identifying broad research priorities of researchers and the cystic fibrosis community. J Cys Fibros 2018;18(3):375-377

${ }^{18}$ https://www.cff.org/Research/Research-Into-the-Disease/Research-into-CFComplications/Infection-Research-Initiative/ Accessed September 15, 2019

${ }^{19}$ Kalaitzis IS, Rowbotham NJ, Smith SJ, Smyth AR. Do current clinical trials in cystic fibrosis match the priorities of patients and clinicians? A systematic review. J Cys Fibros 2020;19(1):26-33

${ }^{20}$ Hurley MN, Ariff AHA, Bertenshaw C, Bhatt J, Smyth AR. Results of antibiotic susceptibility testing do not influence clinical outcome in children with cystic fibrosis. J Cys Fibros 2012; 11(4): 288-92

${ }^{21}$ Aaron SD, Ferris W, Ramotar K, Vandemheen K, Chan F, et al. Combination antibiotic susceptibilities of planktonic, adherent, and biofilm-grown Pseudomonas aeruginosa isolates cultured from sputa of adults with cystic fibrosis. J Clin Microbiol 2002; 40(11): 4172-9

22 Johnson C, Butler SM, Konstan MW, Morgan W, Wohl ME. Factors influencing outcomes in cystic fibrosis: a center-based analysis. Chest 2003;123:20-7.

${ }^{23}$ MacNeill SJ, Pierotti L, Mohammed MA, Wildman M, Boote J, et al. Identifying exceptional cystic fibrosis care services: combining statistical process control with focus groups.

Southampton (UK): NIHR Journals Library; 2019 Feb.

${ }^{24}$ Rowbotham NJ, Smyth AR. Patient engagement to prioritise CF research: Inclusive or selective? J Cyst Fibros 2019;18:307-308

${ }^{25}$ Muhlebach MS, Beckett V, Popowitch E, Miller MB, Mayer-Hamblett N, et al. Microbiological efficacy of early MRSA treatment in cystic fibrosis in a randomized controlled trial. Thorax 2017;72(4):318-326

${ }^{26}$ Langton-Hewer SC, Smyth AR. Antibiotic strategies for eradicating Pseudomonas aeruginosa in people with cystic fibrosis. Cochrane Database Syst Rev 2017: Issue 4

${ }^{2727}$ West N, Beckett V, Raksha J, Sanders D, Nick J, et al. Standardized treatment of pulmonary exacerbations (STOP) study. J Cyst Fibrosis 2017;16(5):600-606

${ }^{28}$ Heltshe SL, West NE, VanDevanter DR, Sanders DB, Beckett VV, et al. Study design considerations for the Standardized Treatment of Pulmonary Exacerbations 2 (STOP2): A trial to compare intravenous antibiotic treatment durations in CF. Contemp Clin Trials. 2018 Jan;64:35-40 
${ }^{29}$ Saiman L, Siegel JD, LiPuma JJ, Brown RF, Bryson EA, et al. Infection prevention and control guideline for cystic fibrosis: 2013 update. Infect Control Hosp Epidemiol 2014; 35 Suppl 1: S1- S67

${ }^{30}$ Moskowitz SM, Emerson JC, McNamara S, Shell RD, Orenstein DM, Rosenbluth D, et al. Randomized trial of biofilm testing to select antibiotics for cystic fibrosis airway infection.

Pediatric Pulmonology 2011;46(2):184-92.

${ }^{31}$ Yau YCW, Ratjen F, Tullis E, Wilcox P, Freitag A, Chilvers M, et al. Online supplementary tables from "Randomized controlled trial of biofilm antimicrobial susceptibility testing in cystic fibrosis patients". J Cyst Fibrosis 2015;14:1-4 online. 